\title{
THE DOS AND DON'TS OF DISTILLATION COLUMN CONTROL
}

\author{
S. Skogestad* \\ Department of Chemical Engineering, Norwegian University of Science and Technology, \\ Trondheim, Norway.
}

Abstract: The paper discusses distillation column control within the general framework of plantwide control. In addition, it aims at providing simple recommendations to assist the engineer in designing control systems for distillation columns. The standard LV-configuration for level control combined with a fast temperature loop is recommended for most columns.

Keywords: configuration selection; temperature location; plantwide control; self-optimizing control; process control; survey.

\section{INTRODUCTION}

Distillation control has been extensively studied over the last 60 years, and most of the dos and don'ts presented in this paper can be found in the existing literature. In particular, the excellent book by Rademaker et al. (1975) contains a lot of useful recommendations and insights. The problem for the 'user' (the engineer) is to find her (or his) way through a bewildering literature (to which I also have made contributions). Important issues (and decisions) that need to be addressed by the engineer are related to the following three problems:

*Correspondence to: Professor S. Skogestad, Department of Chemical Engineering, Norwegian University of Science and Technology, N-7491 Trondheim, Norway. E-mail:skoge@chemeng. ntnu.no

DOI: $10.1205 /$ cherd06133

0263-8762/07/

$\$ 30.00+0.00$

Chemical Engineering

Research and Design

Trans IChemE,

Part A, January 2007

(C) 2007 Institution

of Chemical Engineers
(1) The configuration problem: How should pressure and level be controlled, and more specifically, what is the 'configuration' defined as the two remaining degrees of freedom, after having closed the pressure and level loops? For example, should one use the standard LV-configuration (Figure 1), where condensation flow $V_{T}$ controls pressure $p$, distillate flow $D$ controls condenser level and bottoms flow $B$ controls reboiler level, such that reflux $L$ and boilup $V$ remain as degrees of freedom for composition control. Alternatively, should one use a 'material balance' configuration (DV, LB), a ratio configuration $(L / D V ; L / D V / B$, and so on.)-or maybe even the seemingly 'unworkable' DB-configuration?

(2) The temperature control problem: Should one close a temperature loop, and where should the temperature sensor be located?

(3) The composition control problem (primary controlled variables): Should two, one or no compositions be controlled?
The main objectives of this work are two-fold:

(1) Derive control strategies for distillation columns using a systematic procedure. The general procedure for plantwide control of Skogestad (2004) is used here.

(2) From this derive simple recommendations that apply to distillation column control.

Is the latter possible? Luyben (2006) has his doubts: 'There are many different types of distillation columns and many different types of control structures. The selection of the "best" control structure is not as simple as some papers claim. Factors that influence the selection include volatilities, product purities, reflux ratio, column pressure, cost of energy, column size and composition of the feed.'

Shinskey (1984) made an effort to systematize the configuration problem using the steady-state RGA. It generated a lot of interest at the time and provides useful insights, but unfortunately the steady-state RGA is generally not a very useful tool for feedback control (e.g., Skogestad and Postlethwaite, 2005). For example, the DB-configuration seems impossible from an RGA analysis because of infinite steady-state RGA-elements, but it is workable in practice for dynamic reasons (Finco et al., 1989). The RGA also fails to take into account other important issues, such as disturbances, the overall control objectives (economics) and closing of inner loops such as for temperature.

The paper starts with an overview of the general procedure for plantwide control, and then applies it to the three distillation problems introduced above. Simple recommendations are given, whenever possible. 


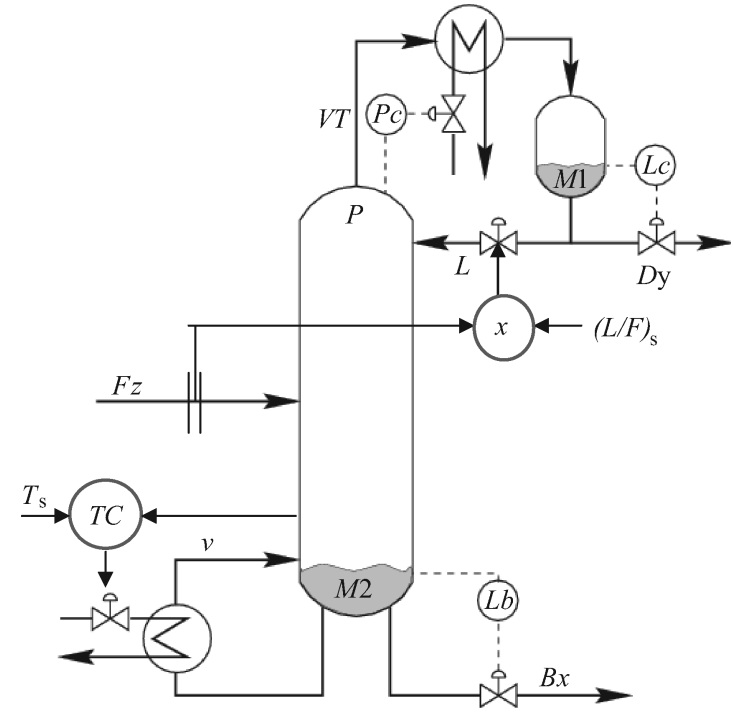

Figure 1. Distillation column controlled with $L V$-configuration. On top of this is added a bottom section temperature controller using $V$, and an $L / F$ feedforward loop. ${ }^{1}$

\section{GENERAL PLANTWIDE CONTROL PROCEDURE}

In this section, the general plantwide control procedure of Skogestad (2004) is summarized. The procedure is applied to distillation control in the subsequent sections. With reference to the control hierarchy in Figure 2, the two main steps are (1) a top-down mainly steady-state (economic) analysis to identify degrees of freedom and corresponding primary controlled variables $y_{1}$, and (2) a bottom-up mostly dynamic analysis to identify the structure of the regulatory control layer including choice of secondary controlled variables $y_{2}$.

\section{Step 1: 'Top-Down' Steady-State Approach Where the Main Objective is to Consider Optimal Plantwide Operation and from this Identify Primary Controlled Variables (Denoted $y_{1}$ or $c$ )}

A steady-state analysis is sufficient provided the plant economics depend primarily on the steady state. First, one needs to quantify the number of steady-state degrees of freedom. This is an important number because it equals the number of primary controlled variables that we need to select.

Second, the steady-state operation (economics) should be optimized with respect to the degrees of freedom for expected disturbances, using a nonlinear steady-state plant model. This requires that one identifies a scalar cost function $J$ to be minimized. Typically, an economic cost function is used:

$$
\begin{aligned}
J= & \text { cost of feed }- \text { value of products } \\
& + \text { cost of energy }
\end{aligned}
$$

Other operational objectives are included as constraints. The cost $J$ is then minimized with respect to the steady-state

${ }^{1}$ Feedforward $L / F$ : The measurement of $F$ is usually send through a first-order lag to improve the dynamic response.

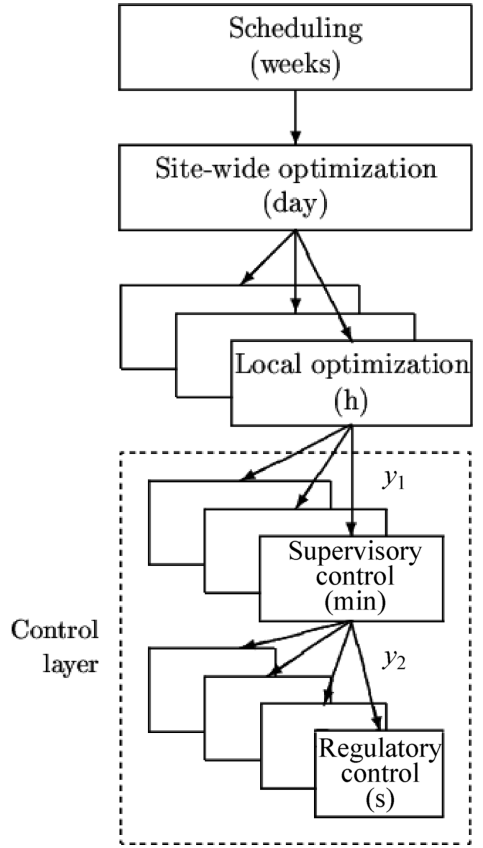

Figure 2. Typical control hierarchy in chemical plant.

degrees of freedom and a key point is to identify the active constraints, because these must be controlled to achieve optimal operation. For the remaining unconstrained degrees of freedom (inputs $\boldsymbol{u}$ ), the objective is to find sets of 'self-optimizing variables', which have the property that near-optimal operation is achieved when these variables are fixed at constant setpoints.

Two approaches to identify self-optimizing (unconstrained) controlled variables for distillation are:

(1) Look for variables with small optimal variation in response to disturbances (Luyben, 1975).

(2) Look for variables with large steady-state sensitivity (Tolliver and McCune, 1980), or more generally, with a large gain in terms of the minimum singular value $\mathbf{S}(\mathbf{G})$ from the inputs $\boldsymbol{u}$ (unconstrained steady-state degrees of freedom) to the candidate controlled outputs $c$ (Moore, 1992).

The two approaches may yield conflicting results, but Skogestad (2000) and Halvorsen et al. (2003) showed how they can be combined into a single rule-the scaled 'maximum gain' (minimum singular value) rule:

- Look for sets controlled variables $c$ that maximize the gain (the minimum singular value) of the scaled steady-state gain matrix, $\sigma\left(\boldsymbol{G}^{\prime} \phi\right)$, where $G^{\prime}=S_{1} G S_{2}$.

The correct choice for the input 'scaling' is $S_{2}=J_{\mathrm{uu}}^{-1 / 2}$ where $J_{\mathrm{uu}}$ is the Hessian matrix (second derivative of the cost with respect to the inputs). Although independent of the choice for $c, S_{2}=J_{u u}^{-1 / 2}$ must nevertheless be included in the multivariable case because it may amplify different directions in the gain matrix $G$ for $c$. The effect of the cost function and the disturbances, enter indirectly into the diagonal output scaling matrix, $S_{1}=\operatorname{diag}\left\{1 / \operatorname{span}\left(c_{\mathrm{i}}\right)\right\}$. Here $\operatorname{span}\left(c_{\mathrm{i}}\right)$ is the 
expected variation in $c_{\mathrm{i}}$ :

$$
\begin{aligned}
\operatorname{Span}\left(c_{\mathrm{i}}\right)= & \mid \text { optimal variation in } c_{\mathrm{i}} \mid \\
& +\mid \text { implementation error for } c_{\mathrm{i}} \mid
\end{aligned}
$$

The optimal variation in $c_{\mathrm{i}}$ is due to disturbances $d$, may be obtained by optimizing for various disturbances using a steady-state model. The steady-state implementation error is often the same as the measurement error. For example, if we are considering temperatures as candidate controlled variables, then a typical implementation error is $0.5 \mathrm{C}$. In the scalar case, the minimum singular value is simply the gain $\left|G^{\prime}\right|$, and here the factor $\left|J_{\text {uu }}\right|$ does not matter as it will have the same effect for all choices for $c$. Therefore, for the scalar case we may rank the alternatives based on maximizing $|G| / \operatorname{span}(c)$.

Note that only steady-state information is needed for this analysis and $G^{\prime}$ may be obtained, for example, using a commercial process simulator. One first needs to find the nominal optimum, and then make small perturbations in the unconstrained inputs (to obtain $G$ for the various choices for $c$ ), reoptimize for small perturbations in the disturbances $d$ (to obtain the optimal variation that enters in $S_{1}$ ), and reoptimize for small perturbations in $u$ (to obtain $J_{u u}$ that enters in $S_{2}$ ).

\section{Step 2: Bottom-Up Identification of a Simple Regulatory ('Stabilizing') Control Layer}

The main objective of the regulatory layer is to 'stabilize' the plant. The word 'stabilize' is put in quotes, because it does not refer to its meaning only in the mathematical sense, but in the more practical sense of 'avoiding drift'. More specifically, we here identify 'extra' secondary controlled variables (denoted $y_{2}$ ) and pair these with manipulated inputs (denoted $u_{2}$ ). The main idea is that control of the variables $y_{2}$ stabilizes the plant and avoids drift. Typical secondary variables include liquid levels, pressures in key units, some temperatures (e.g., in reactors and distillation columns) and flows. The upper layer uses the setpoints $y_{2 s}$ as manipulated variables, and when selecting $y_{2}$ one should also avoid introducing unnecessary control problems as seen from the upper layer. This results in a hierarchical control structure, with the fastest loop (typically the flow and pressure loops) at the bottom of the hierarchy. The number of possible control structures is usually extremely large, so in this part of the procedure one aims at obtaining a good but not necessarily optimal structure.

Some guidelines for selecting secondary controlled variables $y_{2}$ in the regulatory control layer:

(1) The 'maximum gain rule' is useful also for selecting $y_{2}$, but note that the gain should be evaluated at the frequency of the layer above. Often the upper layer is relatively slow and then a steady-state analysis may be sufficient (similar the one used when selecting $y_{1}$ ).

(2) Since the regulatory layer is at the bottom of the hierarchy it is important that in does not fail. Therefore, one should avoid using 'unreliable' measurements.

(3) For dynamic reasons one should avoid variables $y_{2}$ with a large (effective) time delay. This, together with the issue of reliability, usually excludes using compositions as secondary controlled variables $y_{2}$.

(4) To avoid unnecessary cascades and reduce complexity, control primary variables $y_{1}$ in the regulatory layer (i.e., choose $y_{2}=y_{1}$ ), provided guidelines 2 and 3 are met.

The selected secondary outputs $y_{2}$ also need to be 'paired' with manipulated inputs $u_{2}$. Some guidelines for selecting $u_{2}$ in the regulatory control layer:

(1) To avoid failure of the regulatory control layer, avoid variables $u_{2}$ that may saturate (if one uses a variable that may saturate, then it should be monitored and 'reset' using extra degrees of freedom in the upper control layer).

(2) Avoid variables $u_{2}$ where (frequent) changes are undesirable, for example, because they disturb other parts of the process.

(3) Prefer pairing on variables 'close' to each other such that the effective time delay is small.

Eventually, as loops are closed one also needs to consider the controllability of the 'final' control problem which has the primary controlled variables $y_{1}=c$ as outputs and the setpoints to the regulatory control layer $y_{2 s}$ as inputs. In the end, dynamic simulation may be used to check the proposed control structure, but as it is time consuming and requires a dynamic model it should be avoided if possible.

We now apply the two-step procedure to distillation, starting with the selection of primary controlled variables (Step 1).

\section{PRIMARY CONTROLLED VARIABLES FOR DISTILLATION (STEP 1)}

When deriving overall controlled objectives (primary controlled objectives) one should generally take a plantwide perspective and minimize the cost for the overall plant. However, this may be very time consuming, so in practice one usually performs a separate 'local' analysis for the distillation columns based on internal prices. The cost function (1) for a two-product distillation column is typically

$$
\begin{aligned}
J= & p_{\mathrm{F}} F-p_{\mathrm{D}} D-p_{\mathrm{B}} B+p_{\mathrm{Qh}}\left|Q_{\mathrm{h}}\right|+p_{\mathrm{Qc}}\left|Q_{\mathrm{c}}\right| \\
& \approx p_{\mathrm{F}} F-p_{\mathrm{D}} D-p_{\mathrm{B}} B+p_{\mathrm{V}} V
\end{aligned}
$$

where the (internal, 'shadow') prices $p_{\mathrm{i}}$ for the feed $F$ and products $D$ and $B$ should reflect the plantwide setting. The approximation leading to the final expression in (2) applies because typically $\left|Q_{h}\right| \approx\left|Q_{c}\right|$, and we introduce $V=\left|Q_{h}\right| / c$ where the constant $c$ is the heat of vaporization $\left(\mathrm{J} \mathrm{mol}^{-1}\right)$. Then $p_{\mathrm{V}}=c\left(p_{\mathrm{Qh}}+p_{\mathrm{Qc}}\right)$ represents the cost of heating plus cooling.

The cost $J$ in equation (2) should be minimized with respect to the degrees of freedom, subject to satisfying the operational constraints. Typical constraints for distillation columns include:

\section{Purity top product $(D)$ : \\ Purity bottom product $(B)$ : \\ Flow and capacity constraints: \\ Pressure constraint:}

To avoid problems with infeasibility or multiple solutions, the impurity should be in terms of heavy key (HK) component for $D$, and light key (LK) component for $B$. Many columns do not produce final products, and therefore do not have 
purity constraints. However, except for cases where the product is recycled, there are usually indirect constraints imposed by product constraints in downstream units, and these should then be included.

In general, a conventional two-product distillation column has four steady-state degrees of freedom (for example, feedrate, pressure and two column compositions), but unless otherwise stated we assume in this paper that feedrate and pressure are given. More specifically, the feedrate is assumed to be a disturbance, and the pressure should be controlled at a given value. There are then two steady-state degrees of freedom related to product compositions and we want to identify two associated controlled variables.

\section{Composition Control}

Assume that the feedrate $(F)$ and pressure $(p)$ are given, and that there are purity constraints on both products. Should the two degrees of freedom be used to control both compositions ('two-point control')?

To answer this in a systematic way, we need to consider the solution to the optimization problem. In general, we find by minimizing the cost $J$ in (2) that the purity constraint for the most valuable product is always active. The reason is that we should produce as much as possible as the valuable product, or in other words, we should avoid product 'giveaway'. For example, consider separation of methanol and water and assume that the valuable methanol product should contain maximum $2 \%$ water. This constraint is clearly always active, because in order to maximize the production rate we want to put as much water as possible into the methanol product.

However, the purity for the less valuable product constraint is not necessarily active. There are two cases (the term 'energy' used below includes energy usage both for heating and cooling):

- Case 1: If energy is 'expensive' [ $p_{V}$ in (2) sufficiently large] then the purity constraints for the less valuable product is active because it costs energy to overpurify.

- Case 2: If energy is sufficiently 'cheap' ( $p_{\vee}$ sufficiently small), then in order to reduce the loss of the valuable product, it will be optimal to overpurify the less valuable product (that is, its purity constraint is not active). There are here again two cases.

- Case 2a (energy moderately cheap): Unconstrained optimum where $V$ is increased until the point where there is an optimal balance (trade-off) between the cost of increased energy usage $(V)$, and the benefit of increased yield of the valuable product

- Case $2 b$ (energy very cheap): Constrained optimum where it is optimal to increase the energy $(V)$ until a capacity constraint is reached (e.g., $V$ is at its maximum or the column approaches flooding).

In general, we should for optimal operation control the active constraints. A deviation from an active constraint is denoted 'back-off' and always has an economic penalty. The control implications are:

- Case 1 ('expensive' energy): Use 'two-point' control with both products at their purity constraints.

- Case 2a ('moderately cheap' energy where capacity constraint is not reached): The valuable product should be controlled at its purity constraint and in addition one should control a 'self-optimizing' variable which, when kept constant, provides a good trade-off between energy costs and increased yield. In most cases a good self-optimizing variable is the purity of the less valuable product. Thus, 'two-point' control is usually a good policy also in this case, but note that the less valuable product is overpurified, so its setpoint needs to be found by optimization.

- Case 2b ('cheap' energy where capacity constraint is reached): Use 'one-point' control with the valuable product at its purity constraint and $V$ increased until the column reaches its capacity constraint. Note that the cheap product is overpurified.

In summary, we find that 'two-point' control is a good control policy in many cases, but 'one-point' control is optimal if energy is sufficiently cheap such that one wants to operate with maximum energy usage.

\section{Remark}

The above discussion on composition control has only concerned itself with minimizing the steady-state cost $\mathrm{J}$. In addition, there are dynamic and controllability considerations and these generally favour overpurifying the products. The reason is simply that a 'back-off' from the purity specifications makes composition control simpler. Overpurification generally requires more energy, but for columns with many stages (relative to the required separation) the optimum in $J$ is usually very flat, so the additional cost may be very small. Before deciding on the composition setpoints it is therefore recommended to perform a sensitivity analysis for the cost $J$ with the product purity as a degree of freedom.

\section{STABILIZING CONTROL LAYER FOR DISTILLATION (STEP 2)}

With a given feedrate, a standard two-product distillation column has five dynamic control degrees of freedom (manipulated variables; inputs $u$ ). These are the following five flows:

$$
\begin{aligned}
u= & \operatorname{reflux} L, \text { boilup } V \text {, top product (distillate) } D, \\
& \text { bottoms product } B \text {, overhead vapor } V_{T}
\end{aligned}
$$

In practice, $V$ is often manipulated indirectly by the heat input $\left(Q_{h}\right)$, and $V_{T}$ by the cooling $\left(Q_{c}\right)$. In terms of stabilization, we need to stabilize the two integrating modes associated with the liquid levels (masses) in the condenser and reboiler $\left(M_{\mathrm{D}}\right.$ and $\left.M_{\mathrm{B}}\right)$ In addition, for 'stable' operation it is generally important to have tight control of pressure $(p)$, at least in the short time scale (Shinskey, 1984).

However, even with these three variables $\left(M_{\mathrm{D}}, M_{\mathrm{B}}, p\right)$ controlled, the distillation column remains (practically) unstable with a slowly drifting composition profile (in fact, this mode in some cases even become truly unstable ${ }^{2}$ ). To understand this, one may view the distillation column as a 'tank' with light component in the top part and heavy component in the bottom part. The 'tank level' (column profile) needs to be

${ }^{2}$ We may have instability with the $L V$-configuration when separating components with different molecular weights (e.g., methanol and propanol), because a constant mass reflux may give an 'unstable' molar reflux due to a positive composition feedback (Jacobsen and Skogestad, 1994). 
controlled in order to avoid that it drifts out of the column, resulting in breakthrough of light component in the top or heavy component in the bottom.

To stabilize the column profile we must use feedback control as feedforward control cannot change the dynamics and will eventually give drift. A simple measure of the profile location is a temperature measurement $(T)$ inside the column, so a practical solution is to use temperature feedback. This feedback loop should be fast, because it takes a relatively short time for a disturbance to cause a significant composition change at the column ends. As for level control, a simple proportional controller may be used, or a PI-controller with a relatively large integral time.

In summary, we have found that the following variables should be controlled in the stabilizing (regulatory) control layer:

$$
y_{2}=M_{\mathrm{D}}, M_{\mathrm{B}}, p, T
$$

One degree of freedom (flow) remains unused after closing these loops. In addition, the upper layer may manipulate the four setpoints for $y_{2}$. However, note that the setpoints for $M_{\mathrm{D}}$ and $M_{\mathrm{B}}$ have no steady-state effect. The setpoint for $p$ has some (but generally not a significant) steady-state effect, although it is often optimal to minimize $p$ on the long time scale (at steady-state) in order to improve the relative volatility (Shinskey, 1984). In general, the setpoint for $T$ has a quite large steady-state effect on product compositions and it is usually manipulated by an upper layer composition controller. However, because the upper layer usually operates on a quite long time scale, we generally want to select a temperature location such that we achieve indirect composition control (with a constant temperature setpoint), and this is further discussed later.

The selected secondary outputs $y_{2}$ need to be 'paired' with manipulated variables (inputs) $u_{2}$. In this paper, we assume that pressure is controlled using $V_{\mathrm{T}}$ (cooling), although there are other possibilities. The choice of inputs for the other variables is discussed in more detail below:

- Control configuration: Addresses the issue of which inputs $u_{2}$ to use for level control (or actually, which inputs $u_{1}$ (flows) that remain for control of $y_{1}$ after the pressure and level control loops have been closed).

- Temperature control: Addresses the location of the temperature measurement and which input to use for temperature control (or actually, which input (flow) that remains as an 'unused' degree of freedom (fixed on a fast time scale) after the temperature loop has been closed).

\section{Remark}

Throughout this paper the feedrate $F$ is assumed to be given (i.e., $F$ is a disturbance). However, for columns that produce 'on demand', $F$ is a degree of freedom (input), and instead $D$ or $B$ becomes a disturbance. How does this change the analysis below? With given pressure, the number of steady-state degrees freedom is till two. If $B$ is given (a disturbance) and $F$ is liquid (which it usually is), then one may simply replace $B$ by $F$; for example, $F$ is frequently used for reboiler level control. If $D$ is given (a disturbance) and $F$ is liquid, then it is not quite as simple, because $F$ cannot take the role of $D$. Specifically, if $F$ is liquid then it cannot be used for condenser level control, which leaves $L$ or $V$ as candidates for condenser level control, and ' $L V$ '-style configuration can not be used. Such cases will require a more detailed analysis.

\section{CONTROL CONFIGURATION (LEVEL CONTROL)}

The term control 'configuration' for distillation columns usually refers to the two combinations of the four flows $L$, $V, D$ and $B$ that remain (unused) as degrees of freedom (inputs) after the level loops have been closed. For example, in Figure 1 we use the two product flows $D$ and $B$ to control condenser and reboiler level, respectively, and (before we add the feedforward block to get $L / F$ and the feedback temperature loop), reflux $L$ and boilup $V$ remain as degrees of freedom-this is therefore called the $L V$-configuration. The $L V$-configuration is the most common or 'conventional' choice. Another common configuration is the $D V$-configuration, where $L$ rather than $D$ is used to control condenser level. Changing around the level control in the bottom gives the $L B$ configuration. The $D V$ - and $L B$-configurations are known as 'material balance configurations' because the direct handle on $D$ or $B$ directly adjusts the material balance split for the column. Changing around the level control in both ends gives the $D B$-configuration with a direct handle on both $D$ and $B$. This seems unworkable because of the steady-state material balance $D+B=F$, but it is actually workable in practice (Finco et al., 1989) for dynamic reasons (Skogestad et al., 1990). Levels may also be controlled such that ratios remain as degrees of freedom, for example the $L / D V$ - and $L / D V / B$-configurations.

Many books (Shinskey, 1984) and papers, including several of my own (e.g., Skogestad and Morari, 1987), have been written on the merits of the various configurations, but it is probably safe to say that the importance of the choice of configuration (level control scheme) has been overemphasized. The main reason is that the distillation column, even after the two level loops (and pressure loop) have been closed, is 'practically unstable' with a drifting composition profile. To avoid this drift, one needs to close one more loop, typically a relatively fast temperature loop (often faster than the level control loops). This fast loop will again influence the level control. Thus, an analysis of the various configurations (level control schemes), without including a temperature or quality loop, is generally of limited usefulness.

\section{Difference Between Control Configurations Without a Temperature Loop}

Although we just stated that it is of limited usefulness, we will first look at the difference between the various 'pure' configurations (without a temperature loop). One reason is that this problem has been widely studied and discussed in the distillation literature. Over the years, the distillation experts have disagreed strongly on what is the 'best' configuration. The reason for the controversy is mainly that the various experts put varying emphasis on the following possibly conflicting issues:

(1) Level control by itself (emphasized e.g., by Buckley et al., 1985). 
(2) Interaction of level control (in particular the level control tuning) with the remaining composition control problem (Skogestad, 1997).

(3) 'Self-regulation' in terms of disturbance rejection (emphasized e.g., by Skogestad and Morari, 1987).

(4) Remaining two-point control problem in terms of steadystate interactions (emphasized e.g., by Shinskey, 1984).

\section{Level control}

If we look at liquid level control by itself, then it is quite clear that one generally should use the largest flow to control level. The reason is that it is then less likely that the flow will saturate, which as noted previously should be avoided in the lower layers of the control hierarchy. For example, consider control of top level (reflux drum) where one issue is whether to use $L$ or $D$ as an input. The 'largest flow' rule gives that one should use distillate $D$ (the 'conventional choice') if $L / D<1$, and reflux $L$ for higher reflux columns with $L / D>1$.

Partly based on this reasoning, Liptak (2006, Chapter 8.19) recommends for top level control to use $D$ for $L / D<0.5$ (low reflux ratio), and $L$ for $L / D>6$ (high reflux ratio). For intermediate reflux ratios either $L$ or $D$ may be used. Thus, the $L V$-configuration is not recommended for $L / D>6$. Similar arguments apply to the bottom level, that is, the standard scheme with $B$ for bottom level control is not recommended if $V / B$ is large $(>6)$. However, as discussed in more detail below, these recommendations do not apply when a temperature loop is included, because of the 'indirect' level control resulting from the temperature loop.

\section{Interaction between level and composition control}

It is generally desirable that level control and column (composition) control are decoupled. That is, retuning of a level controller should not affect the remaining control system. This clearly favors the $L V$-configuration (where $D$ and $B$ are used for level control) because $D$ and $B$ have by themselves no effect on the rest of the column.

For example, assume that $L$ is used for top level control (e.g., $D V$-configuration). The remaining flow $D$ in the top can then affect the column only indirectly through the action of the level controller which manipulates $L$. The top level controller then has to be tightly tuned to avoid that the response from $D$ to compositions is delayed and depends on the level tuning. Furthermore, with tight level control, one is not really making use of the level as a 'buffer' and one might as well eliminate the reflux drum. On the other hand, with the $L V$-configuration (where $D$ is used for top level control), the remaining flow $L$ has a direct effect on the column and the level control tuning has no (or negligible) effect on the composition response for $L$.

\section{Disturbance rejection}

The $L V$-configuration generally has poor self-regulation for disturbances in $F, V, L$ and in feed enthalpy (Skogestad and Morari, 1987). That is, with only the level loops closed using $D$ and $B$, the composition response is very sensitive to these disturbances. The $D V$ - or $L B$-configurations generally behave better in this respect, because disturbances in $V, L$ and feed enthalpy are kept inside the column and do not affect the external flows (because $D$ is constant). The double ratio configuration $L / D \quad V / B$ has even better self-regulating properties, especially for columns with large internal flows (large $L / D$ and $V / B$ ). These conclusions are supported by the relative composition deviations $\Delta X$ computed for various configurations for a wide range of distillation columns (Hori and Skogestad, 2006); for example see the data for 'column A' given in the left two columns in Table 1.

\section{Remaining composition control problem}

With the $L V$-configuration, the remaining composition problem is generally interactive and ill-conditioned, especially at steady-state and for high-purity columns. This is easily explained because an increase in $L$ (with $V$ constant) has essentially the opposite effect on composition of an increase in $V$ (with $L$ constant). Thus, the two inputs counteract each other and the process is strongly interactive. This can be quantified by computing the relative gain array (RGA). The steady-state RGA (more precisely, its 1,1-element, which preferably should be close to 1) for various configurations for 'column A' are (Skogestad and Morari, 1987):

$$
\begin{aligned}
& L / D-V / B: 3.22, \quad L-B: 0.56, \quad D-V: 0.45, \\
& L / D-V: 5.85, \quad L-V: 35.1, \quad D-B: \infty
\end{aligned}
$$

Note that the steady-state RGA is very large for the $L V$ configuration and that it is infinite for the DB-configuration. This has led many authors (e.g., Shinskey, 1984) to conclude that the $D B$-configuration is infeasible, but this conclusion is incorrect. Indeed, with a given feed flow, $D$ and $B$ can not be changed independently at steady-state because of the constraint $D+B=F$, but it is possible to make independent changes dynamically because of the holdup in the column. Similarly, the $L V$-configuration is much less interactive dynamically than the large steady-state RGA-value of 35.1 indicates. This reason is that an increase in reflux $L$ will immediately influence the composition in the top of the column, whereas it takes some time ( $q_{\mathrm{L}}$; typically a few minutes) to 'move down' the column and influence the bottom composition. This can be more clearly seen from a frequency-dependent RGA-plot (not shown in this paper),

Table 1. Relative steady-state composition deviation $D X=$ $\sqrt{\left(\left(x_{\text {top }}^{\mathrm{H}}-x_{\mathrm{top}, \mathrm{s}}^{\mathrm{H}}\right) / x_{\text {top }, \mathrm{s}}^{\mathrm{H}}\right)^{2}+\left(\left(x_{\mathrm{btm}}^{\mathrm{L}}-x_{\mathrm{btm}, \mathrm{s}}^{\mathrm{L}}\right) / x_{\mathrm{btm}, \mathrm{s}}^{\mathrm{L}}\right)^{2}}$ for sum of disturbances in feed rate, feed composition, feed enthalpy and implementation error for some control structures (Hori and Skogestad, 2006).

\begin{tabular}{lccc}
\hline $\begin{array}{l}\text { Fixed flows } \\
\text { (configuration) }\end{array}$ & $\Delta X$ & $\begin{array}{c}\text { Fixed } \\
\text { flow and } T\end{array}$ & $\Delta X$ \\
\hline$L / D-V / B$ & 15.8 & $T_{12}-T_{30}$ & 0.53 \\
$L / F-V / B$ & 18.6 & $T_{15}-L / F$ & 0.92 \\
$L-B$ & 21.1 & $T_{16}-V / F$ & 1.15 \\
$D-V$ & 21.2 & $T_{19}-L$ & 1.22 \\
$L / D-V$ & 23.1 & $T_{15}-L / D$ & 1.32 \\
$L-V$ & 63.4 & $T_{22}-V$ & 1.47 \\
& & $T_{24}-V / B$ & 1.71 \\
& & $T_{39}-B$ & 29.9
\end{tabular}

Data column A: Binary separation of ideal mixture with relative volatility 1.5; column with 40 stages, feed stage at 21 (counted from bottom); 0.01 mole fraction impurity in both products. 
where $\bar{\equiv}$ 1-element approaches 1 at frequencies above about $1 / q_{\mathrm{L}}\left(\mathrm{rad} \mathrm{s}^{-1}\right.$, both for the $L V$ - and $D B$-configurations. Stated in simple terms, it is possible to 'decouple' the composition control in the two column ends, provided the closed-loop response time, at least in one end, is about $q_{\mathrm{L}}$ or less. However, this decoupling is usually not possible to achieve if we have only composition control (with no inner temperature loop) because of the large measurement delay often associated with measuring composition (typically with a gas chromatograph) that forces one to control at a slower time scale. Thus, for the case with no inner temperature loop, a steady-state RGA-analysis may indeed be a useful tool for evaluating the interactions with alternative configurations.

\section{Summary: recommendations for (flow) configurations without closing a temperature loop}

Shinskey (1984) suggests using the steady-state RGA as a 'unifying' measure to summarize all the conclusions from the four issues listed above. In fact the correlation (which is mainly empirical) is quite good. Shinskey's rule (although he does not express it explicitly) is to prefer configurations with a steady-state RGA in the range from about 0.9 to 4 (Shinskey, 1984, table 5.2). The steady-state RGA is also recommended as a useful tool in more recent control engineering handbooks (Liptak, 2006, Chapter 8.20). For columns where the top product is purest (compared to the bottom product) the RGA favours the $L B$-configuration (because the RGA is then close to 1), whereas the DV-configuration is favored for a pure bottom product. For cases where the purities of the two products are similar, the RGA generally favors the double ratio $(L / D V / B)$-configuration. Shinskey only recommends the $L V$-configuration for 'easy' separations with (1) a low reflux ratio $L / D$ and (2) at least one relatively unpure product (in the range of a few percent impurities). However, note that this recommendation is for the case with composition control in both ends and with no 'fast' temperature loop closed. As already argued and discussed in more detail below, one should close a temperature loop, and in this case the recommendations of Shinskey do not apply.

\section{Difference Between Configurations with a Temperature Loop}

The temperature control problem is considered in more detail in the next section, but note already now that all the four issues discussed above change in favour of the $L V$-configuration when a temperature loop is closed:

(1) Closing a temperature loop gives 'indirect' level control, and levels may be controlled with $D$ and $B(L V-$ configuration) even for high reflux columns.

(2) Interaction of level control with remaining composition control is negligible with the $L V$-configuration.

(3) 'Self-regulation' in terms of disturbance rejection (which is unfavorable for the $L V$-configuration) is much better when a temperature loop is closed (see right two columns in Table 1).

(4) With a sufficiently fast temperature loop, there is almost no two-way interaction in the remaining two-point control problem, that is, the steady-state RGA approaches 1, even for the $L V$-configuration.

\section{TEMPERATURE CONTROL}

Temperature control is very common in industrial practice, because, as just mentioned, there are a number of benefits of closing a reasonably fast temperature loop:

(1) Stabilizes the column composition profile (and thus keeps disturbances within the column).

(2) Gives indirect level control: Reduces the need for level control (as a result of benefit 1).

(3) Gives indirect composition control: Strongly reduces disturbance sensitivity.

(4) Makes the remaining composition problem less interactive (e.g., in terms of the RGA) and thus makes it possible to have good two-point composition control.

(5) Makes the column behave more linearly (as a result of benefits 1 and 2).

Each benefit is discussed in more detail below, and we end the section with a discussion on where to place the temperature sensor.

\section{Stabilization of Column Profile}

Temperature control provides a simple way of stabilizing the column composition profile. This is required, because, as already noted, even with the level and pressure loops closed, a distillation column is 'practically unstable' with an almost integrating mode related to composition.

\section{Indirect Level Control}

A temperature controller provides indirect level control, both in the top and in the bottom. To understand better this benefit, consider a column with large internal flows (say, $V=10 \mathrm{~mol} \mathrm{~s}^{-1}, L=9.5 \mathrm{~mol} \mathrm{~s}^{-1}$ and $D=B=0.5 \mathrm{~mol} \mathrm{~s}^{-1}$ ) controlled with the $L V$-configuration. Without any temperature loop, the two remaining inputs are $L$ and $V$. Even though $V$ is an input, there are often quite large additional disturbances in the boilup $V$, for example, because of varying pressure in the steam used for generating $V$. In any case, assume there is a disturbance such that $V$ decreases from $10 \mathrm{~mol} \mathrm{~s}^{-1}$ to $9 \mathrm{~mol} \mathrm{~s}^{-1}$. For simplicity of the argument, assume feed is liquid, constant molar flows and fixed flows on a molar basis. The vapour flow $V_{\mathrm{T}}$ at the top then also decreases from $10 \mathrm{~mol} \mathrm{~s}^{-1}$ to $9 \mathrm{~mol} \mathrm{~s}^{-1}$, and so does the liquid condensate that enters the condenser. To counteract this, the level controller in the top will decrease $D$ from $0.5 \mathrm{~mol} \mathrm{~s}^{-1}$ to its lower limit of $0 \mathrm{~mol} \mathrm{~s}^{-1}$, but since the reflux $L$ is constant at $9.5 \mathrm{~mol} \mathrm{~s}^{-1}$ there is still an excess of $0.5 \mathrm{~mol} \mathrm{~s}^{-1}$ leaving the reflux drum and it will eventually empty. This is clearly an undesirable situation, and explains why it may be reasonable to not use $D$ for level control when $L / D$ is large, for example when $L / D>6$ (Liptak, 2006).

Now consider the same situation when we have added a temperature controller that manipulates reflux $L$. Initially, the disturbance in $V$ will have a similar effect as before, and the top (reflux drum) level will start dropping. However, the disturbance $V$ will also affect the compositions and temperatures inside the column. The decrease in $V$ will cause less heavy component to go up the column and the temperature will drop. In response to this, the top temperature controller will decrease the reflux $L$, which has the (desired) side effect of counteracting the drop in liquid level in the condenser. Thus, the temperature controller provides indirect level control. The indirect level control effect also applies to the 
reboiler level, and also if the temperature loop is closed using $V$.

As an extreme case of indirect level control, consider a column that removes a very small amount of light impurity in the feed (so $D$ is almost 0 and $L / D$ approaches infinity). According the rule by Liptak (2006, Chapter 8.19), $L$ must be used for top level control, but assume instead that $L$ is used for temperature control and that we have no level control in the top. The light component (a very small amount) will then accumulate slowly in the top by the action of the temperature controller that adjusts $L$, and, depending on the amount of light component in the feed, the top (reflux) drum be emptied for example once a day. This situation with no level control has been experimentally verified in a multivessel distillation column (Wittgens and Skogestad, 2000). This is a closed batch column where the products are collected in vessels along the column without any use of level control. It is the action of the temperature controllers that adjusts the flows out of the vessels and provides indirect level control.

\section{Indirect Composition Control}

A temperature controller provides indirect composition control, provided the temperature is appropriately located.

Assume the objective is to control both product compositions, but we have no 'direct' product composition control or it is slow, for example, because of large measurement delays. A reasonably objective is then to find a control configuration that minimizes the relative steady-state composition deviation ('loss') for the top and bottom product:

$$
D X=\sqrt{\left(\frac{x_{\mathrm{top}}^{\mathrm{H}}-x_{\mathrm{top}, \mathrm{s}}^{\mathrm{H}}}{x_{\mathrm{top}, \mathrm{s}}^{\mathrm{H}}}\right)^{2}+\left(\frac{x_{\mathrm{btm}}^{\mathrm{L}}-x_{\mathrm{btm}, \mathrm{s}}^{\mathrm{L}}}{x_{\mathrm{btm}, \mathrm{s}}^{\mathrm{L}}}\right)^{2}}
$$

The results for a binary distillation example ('column A') are given in Table 1 (for details the reader is referred to Hori and Skogestad, 2006). We here focus on the temperature control structures in the right columns of Table 1. Two degrees of freedom need to be specified (i.e., fixed or controlled). With a given fixed flow (e.g., $L, V, L / F, V / F, L / D$ and $V / B$ ), the results show the best temperature location (found by minimizing $D X$ ). The first thing to note is that the setpoint deviation $D X$ is significantly smaller when we fix one temperature and a flow instead of two flows. Thus, temperature control clearly contributes to give indirect composition control.

The best combination of a temperature with a flow is $T_{15}$ $L / F$ with a relative composition deviation of 0.92 . In practice, this scheme may be implemented using the boilup $V$ to control temperature $T_{15}$ (six stages below the feed) and fixing the reflux-to-feed ratio $L / F$, see Figure 1 . The deviation is somewhat larger when we eliminate the feedforward from $F$ to $L$; fixing $T_{19}-L$ has a deviation of 1.22 . The reason why the difference is not even larger is partly because the implementation error is larger when fixing $L / F$ instead of fixing $L$ (because of the additional uncertainty in measuring $F$ ). Fixing $V / F$ or $V$ has a slightly higher loss (1.15 and 1.47, respectively) than fixing $L / F$ or $L$, and in addition, it requires that we use reflux $L$ to control temperature, which is less favourable dynamically because of the effective delay for reflux to affect stages down the column. Even lower product composition deviations are found when fixing two temperatures. The lowest loss is with one temperature in the middle of each section $\left(T_{12}-T_{30}\right)$ with a deviation of 0.53 .

Similar results are obtained for multicomponent mixtures, except that here it is not necessarily better to fix two temperatures. In fact, for some multicomponent separations fixing two temperatures gives a significantly larger loss than fixing a temperature and a flow (e.g., see the results for the multicomponent splits $A / B$ and $C / D$ in Hori and Skogestad, 2006). The reason is that temperature is a poorer measure of composition for multicomponent mixtures, because of disturbances in feed composition.

In summary, based on a number of case studies (Hori and Skogestad, 2006; Luyben, 2006), fixing $L$ and a temperature (using boilup for temperature control) seems to be a good configuration in most cases. Indeed, this structure (' $L V$-configuration plus a temperature loop using $V$ ) is widely used in industry. Luyben (2006) recommends it only for low to modest reflux ratios, because of the potential problem with the level control, but in the present paper it is argued that the 'indirect level control' effect makes it workable over a much larger range of reflux ratios.

As discussed in more detail below, the temperature should be located at the most sensitive stage ('maximum gain rule') and should generally be located away from the column ends. If tight control of one product is the most important, then one should preferably control a temperature in the same section.

\section{Remaining Composition Control Problem with Temperature Loop Closed}

After having closed the temperature loop, the two inputs left for composition control are the temperature setpoint and flow setpoint, for example, $L_{\mathrm{s}}$ and $T_{\mathrm{s}}$. The composition control problem could be handled using decentralized (single-loop) control, but it is increasingly common to multivariable control, or more specifically model predictive control (MPC). The remaining composition control problem is still quite interactive, which is one reason why multivariable control is attractive, but the interactions are much less severe than for the 'original' LV-problem without the temperature loop. This has been studied by Wolff and Skogestad (1996) who in particular showed that the steady-state RGA may be significantly reduced (e.g., from 35 to less than 2 for 'column A') by closing the temperature loop, provided the temperature loop is sufficiently fast. A fast temperature loop also reduces the 'overshoot' in the response from the inputs to the outputs that may otherwise appear.

\section{Temperature Location}

It has in this paper been argued heavily in favor of implementing a fast 'stabilizing' temperature loop. Two questions are:

(1) Where should the temperature sensor be located?

(2) Which input flow (usually $L$ or $V$; although $D$ and $B$ are also possible) should be used to control it?

These issues have been discussed already, but let us here provide some simple rules [e.g., based on the work of Rademaker (1975), Tolliver and McCune (1980), Moore (1992), Luyben (2006) and Hori and Skogestad (2006)]: 
Rule 1. The location should be within the column rather at the column ends (Tolliver and McCune, 1980). Especially, this is the case at high-purity column ends with a small key component impurity.

Rule 2. Control temperature in the column end where composition control is most important; this is usually for the most valuable product. This minimizes composition variations in the important end, as is confirmed by steady-state analysis (e.g., Hori and Skogestad, 2006) and dynamic simulations (Luyben, 2006).

Rule 3. Locate the temperature where the steady-state sensitivity is large ('maximum gain rule'). The sensitivity (gain $G$ ) is evaluated by perturbing a remaining 'free' input u (e.g., the input used for temperature control) (Tolliver and McCune, 1980). That is, $G=D c / D u$, where $c$ is the candidate controlled temperature. To be rigorous, one should as explained previously, maximize the scaled gain $\left|G^{\prime}\right|=|G|$ / $\operatorname{span}(c)$ (Skogestad, 2000). The span (scaling factor) for temperature on a given stage is the sum of its optimal variation (due to disturbances) and implementation (measurement) error. More generally, with two or more controlled variables $c$ to be selected, one should use the minimum singular value, $\sigma\left(\boldsymbol{G}^{\prime} \phi\right)$, where the scaled gain matrix is $G^{\prime}=$ $\operatorname{diag}\left\{1 / \operatorname{span}\left(c_{\mathrm{i}}\right)\right\} G J_{\mathrm{uu}}^{-1 / 2}$. This is very similar to the SVD method used by Moore (1992), except that Moore did not include scaling.

Rule 4. For dynamic reasons, one should avoid locating a temperature sensor in a region with a small temperature change from one stage to the next (avoid 'flat' temperature profile). This is because the initial dynamic temperature response is proportional to the temperature difference between neighbouring stages (Rademaker et al, 1975, p. 420) (Hori and Skogestad, 2006). If temperature is controlled in a constant temperature region, then this will result in an effective time delay in the response and fast closedloop control is not possible.

Combining rules 3 and 4 gives:

- Control temperature where the (scaled) steady-state gain and the temperature slope are both large (a trade-off may be required in some cases, but not for binary mixtures where rules 3 and 4 give the same location).

Rule 5. Use an input (flow) in the same end as the temperature sensor. There are two reasons:

(1) For $L$ : The temperature loop should be fast, and $L$ should then not be used to control a temperature in the bottom of the column because of the delay $\theta_{\mathrm{L}}$ for a change in liquid to reach the bottom.

(2) For $V$ : There is no delay for the vapour to move up the column, so it is possible to use $V$ to obtain fast temperature control in the top. On the other hand, this is not desirable because it may give strong interactions in the remaining composition control problem. If $V$ is used to control a temperature $T_{\text {top }}$ in the top of the column, $\equiv$ the remaining inputs for composition control are $\sqrt{\mathrm{z} \pi \mathrm{O}}$ $T_{\text {top,s }}$ (setpoint). With single-loop control, the preferred solution is probably to use $T_{\text {top,s }}$ to control top composition and $L$ to control bottom composition, which is clearly an interactive control problem.

Rule 6. Avoid using an input (flow) that may saturate. The reason is that saturation generally should be avoided in stabilizing ('lower') loops, because control is then lost and one is unable to follow setpoint changes from the layer above.

It may be beneficial to use combinations of temperatures, for example, the average temperature in the top section or in the bottom section. This may avoid the sensitivity loss if the temperature sensor moves into a 'flat region', for example, due to feed or product composition changes.

\section{DISCUSSION: TEMPERATURE LOCATION CRITERIA}

Luyben (2006) discusses some alternative criteria (mostly empirically based, including some of the rules listed above) that have been proposed for selecting tray temperatures, and compares them on several case studies. In the following, the theoretical basis for these alternative criteria is discussed, but let us first recall the rigorous scaled maximum gain rule (Halvorsen et al., 2003), which was listed as Rule 3 above:

- Look for sets controlled variables that maximize the minimum singular value of the scaled steady-state gain matrix, $\sigma\left(\boldsymbol{G}^{\prime} \phi\right)$ where $G^{\prime}=S_{1} G S_{2}$.

In the scalar case, the input scaling $S_{2}$ does not matter for selecting the controlled variable (output $c$ ) and the maximum gain rule tells us to look for a controlled variable $c$ that maximizes $|G| / \operatorname{span}(c)$ where $G=d c / d u$ is the steady-state gain, and $\operatorname{span}(c)=\mid$ optimal variation in $c|+|$ implementation error for $c \mid$.

The five criteria listed by Luyben (2006) are next discussed in this context.

1: Slope Criterion: Select the Tray (Location) Where There are Large Changes in Temperature from Tray to Tray

This criterion has no rigorous relationship with the steadystate composition behaviour and should not be used as a single condition to select temperature locations. For example, multicomponent mixtures often display a large change in temperature towards the column end, but this is a poor location of the measurement for indirect composition control (Hori and Skogestad, 2006; Luyben, 2006). On the other hand, as mentioned in the previous section, it can be used as condition to avoid (not select) temperature locations. This is because the temperature slope (change from one stage to the next) is directly related to the initial slope of the dynamic response (Rademaker et al., 1975, p. 420). In summary, one should not use a large slope as a criterion to select the stage, but rather use a small slope as a criterion to avoid a stage.

2: Sensitivity criterion: Find the tray where there is the largest change in temperature for a change in the manipulated variable

This criterion is the same as maximizing the unscaled steady-state gain $G$. This is not by itself a reliable criterion.

3: SVD criterion: Use singular value decomposition analysis

This is the multivariable generalization of maximizing the unscaled gain, $\sigma(\boldsymbol{G})$. As a minor comment, note that Moore (1994) and Luyben (2006) propose to use the 'one-shot' SVD-method which is numerically effective, but does not necessarily give the measurements with the smallest $\sigma(\mathbf{G})$.

4: Invariant temperature criterion: With both the distillate and bottoms purities fixed, change the feed 
composition (disturbance) over the expected range of values. Select the tray where the temperature does not change as feed composition (the disturbance) changes.

This criterion is the same as minimizing the optimal variation (due to disturbances), which is in agreement with the output scaling recommended in Rule 3.

5: Minimum product variability criterion: Choose the tray that produces the smallest change in product purities when it is held constant in the face of the feed composition disturbances.

This is the exact 'brute force' method of Skogestad (2000), except that implementation error is not included. If implementation error were included, then the method would be exact, and it would disfavour temperatures towards the column end (which have a small optimal variation), and some of the problems that Luyben (2006) refers to with the method would be avoided.

In summary,

- criterion 1 should be used to avoid (rather than to select) a temperature location;

- criteria $2 / 3$ and 4 , with the addition of the implementation error, are combined 'optimally' in the scaled 'maximum gain rule': maximize $\sigma\left(\mathbf{G}^{\prime} \phi\right)$ where $G^{\prime}=\operatorname{diag}\left\{1 / \operatorname{span}\left(c_{\mathrm{i}}\right)\right\}$ $G J_{\text {uu }}^{-1 / 2}$ (Halvorsen et al., 2003);

- criterion 5 , with the addition of implementation error, is the exact 'brute force' method presented by Skogestad (2000).

As noted by Luyben (2006), criterion 5 is the most 'fundamental' objective, but it requires more computations, because all candidate temperature locations must be evaluated separately. The more simple and straightforward singular value decomposition analysis (maximize $\sigma(\mathbf{G} \phi)$ ), is therefore recommended to select temperature locations. This agrees with the conclusion of Luyben (2006).

\section{LOGARITHMIC TRANSFORMATIONS}

Let me finally make a point for logarithmic transformations which may prove very useful and reduce the need for retuning and complicated corrections. It is well known that the response of distillation columns, both dynamic and static, is strongly nonlinear. Fortunately, most of the nonlinearity, including that caused by changes in the operating point, may be counteracted using logarithmic transformations (Skogestad, 1997), or equivalently, by scaling with key component impurity (Shinskey, 1984). Especially the benefit is good for the initial part of the dynamic response (Skogestad, 1997) which is important for feedback control purposes. In terms of compositions the logarithmic transformation on a given stage is

$$
X^{\log }=\ln \left(x_{\mathrm{L}} / X_{\mathrm{H}}\right)
$$

where $x_{\mathrm{L}}$ and $x_{\mathrm{H}}$ are the mole fractions of light and heavy key components, respectively. Similarly, the logarithmic temperature on a given stage is

$$
T^{\log }=\ln \left(T_{\mathrm{H}, \text { ref }}-T\right) /\left(T-T_{\mathrm{L}, \text { ref }}\right)
$$

where $T_{\mathrm{L}, \text { ref }}$ is the boiling point of light key component (or some reference temperature measured near the top the column) and $T_{\mathrm{H} \text {,ref }}$ is the boiling point of heavy key component (or some reference temperature measured near the bottom). Usually, we have $X^{\log } \approx T^{\log }$.

\section{OTHER ISSUES}

Many issues have not been discussed in this paper. One is model predictive control (MPC), which is widely used for distillation columns. The main reason for using MPC is usually to handle in a transparent manner cases with changes in the controlled variables, use of extra manipulated variables, and feedforward and multivariable control. One could alternatively achieve the same using simple control elements, including logic switches, split range control, and feedforward and decoupling blocks, but an MPC solution is often simpler to understand and maintain.

In any case, the MPC application is almost always placed on top of the 'basic' control system that has been the focus of this paper. Thus, essentially all the material in this paper, including the importance of controlling the right variables (self-optimizing control), will be relevant when using MPC.

\section{CONCLUSION}

In the introduction two objectives were stated:

(1) Derive control strategies for distillation column using the general procedure for plantwide control of Skogestad (2004).

(2) From this derive simple recommendations that apply to distillation column control.

The first objective has been fulfilled, as the general procedure does indeed apply nicely to distillation column control. The second objective has been reasonably fulfilled. The standard $L V$-configuration is found to be workable for almost all columns, even those with large reflux rates, provided a sufficiently fast inner temperature loop is closed. The temperature loop has many advantages, including providing indirect level control, which makes it possible to use the $L V$-configuration also for high reflux columns. The structure shown in Figure 1, where boilup $V$ is used to control a temperature in the bottom section, is recommended for most columns, but the temperature sensor should be moved to the top section if accurate control of the top product is more important than the bottom product.

Note that this paper has dealt with the 'basic' feedback control system that operates on a quite fast time scale. If one is lucky, this system is sufficient to (indirectly) achieve economic optimal operation ('self-optimizing control'), but in many cases as more 'advanced' supervisory control system is put on top. In the simplest case, this could be a composition control system using two PI controllers, but the trend today is to use multivariable control (model predictive control, MPC) which is able to deal with interactions, feedforward control and constraints in an efficient manner. Composition estimators ('soft sensors') based on temperature measurements are also frequently used, for example based on simple steady-state regression models or a dynamic Kalman filter. Nevertheless, before one starts including such more 'advanced' control strategies, it is important that the basic control system is designed properly, as discussed in this paper.

\section{REFERENCES}

Buckley, P.S., Luyben, W.L. and Shunta, F.S., 1985, Design of Distillation Column Control Systems, (Instrument Society of America, Reserarch Triangle Park, USA) 
Finco, M.V, Luyben, W.L. and Polleck, R.E., 1989, Control of distillation columns with low relative volatility, Ind Eng Chem Res, 28: 76-83.

Halvorsen, I.J., Skogestad, S., Morud, J. and Alstad, V., 2003, Optimal selection of controlled variables, Ind Eng Chem Res, 42: 3273-3284.

Hori, E.S. and Skogestad, S., 2006, Self-optimizing control configurations for two-product distillation columns, Special issue of Chemical Engineering Research and Design from Distillation and Absorption Symposium London, September.

Jacobsen, E.W. and Skogestad, S., 1994, Instability of distillation columns, AlChE J, 40(9): 1466-1478.

Liptak, B.G., 2006, Instrument Engineers' Handbook. Volume II. Process Control and Optimization, 4th edition (CRC Press, Boca Raton, Florida, USA).

Luyben, W.L., 1975, Steady-state energy-conservation aspects of distillation control system design, Ind Eng Chem Fundam, 14: 321.

Luyben, W.L., 2006, Evaluation of criteria for selecting temperature control trays in distillation columns, J Proc Control, 16: 115-134

Moore, C.F., 1992, Selection of controlled and manipulated variables, in Luyben, W.L. (Ed.). Practical Distillation Control (Van Nostrand Reinhold: New York, USA).

Rademaker, O., Rijnsdorp, J.E. and Maarleveld A., 1975, Dynamics and Control of Continuous Distillation Units (Elsevier).

Shinskey, F.G., 1984, Distillation Control, 2nd edition (McGraw-Hill)
Skogestad, S. and Morari, M., 1987, Control configuration selection for distillation columns, AIChE J, 33(10): 1620-1635.

Skogestad, S., Jacobsen, E.W. and Morari, M., 1990, Inadequacy of steady-state analysis for feedback control: Distillate-bottom control of distillation columns, Ind Eng Chem Res, 29(12): 2339-2346.

Skogestad, S., 1997, Dynamics and control of distillation columns-A tutorial introduction, Trans IChemE, 75(Part A): 539-562.

Skogestad, S., 2000, Plantwide control: the search for the selfoptimizing control structure. J Proc Control, 10(5): 487-507.

Skogestad, S., 2004, Control structure design for complete chemical plants, Computers and Chemical Engineering, 28: 219-234.

Skogestad, S. and Postlethwaite, I., 2005, Multivariable Feedback Control, 2nd edition (Wiley: London, UK).

Tolliver, T.L. and McCune, L.C., 1980, Finding the optimum temperature control trays for distillation columns, InTech, 75-80.

Wittgens, B. and Skogestad, S., 2000, Closed operation of multivessel batch distillation: Experimental verification, AlChE J., 46: 1209-1217.

Wolff, E.A. and Skogestad, S., 1996, Temperature cascade control of distillation columns, Ind Eng Chem Res, 35: 475-484.

The manuscript was received 11 August 2006 and accepted for publication after revision 29 November 2006. 